\title{
Reaction-Diffusion based Autonomous Control of Camera Sensor Networks
}

\author{
Katsuya Hyodo \\ Graduate School of \\ Information Science and \\ Technology, Osaka University \\ 1-5 Yamadaoka, Suita, Osaka \\ 565-0871, Japan \\ k-hyodo@ist.osaka- \\ u.ac.jp
}

\author{
Naoki Wakamiya \\ Graduate School of \\ Information Science and \\ Technology, Osaka University \\ 1-5 Yamadaoka, Suita, Osaka \\ 565-0871, Japan \\ wakamiya@ist.osaka- \\ u.ac.jp
}

\author{
Masayuki Murata \\ Graduate School of \\ Information Science and \\ Technology, Osaka University \\ 1-5 Yamadaoka, Suita, Osaka \\ 565-0871, Japan \\ murata@ist.osaka-u.ac.jp
}

\begin{abstract}
Although wireless camera sensor networks are useful and powerful means of monitoring and surveillance, the high volume of video data would cause serious network congestion and degradation of perceived video quality for the limited capacity of wireless communication. In this paper, we propose an autonomous control mechanism of video coding rate, where each node autonomously determines its coding rate based on information obtained through local communication. For this purpose, we applied a reaction-diffusion model, which explains the emergence of variety of animal coat patterns. A spot pattern of video coding rate, which is centered at the target position and spreads towards the moving direction, is generated through local interactions among neighboring nodes. Simulation results show the effectiveness of our proposal.
\end{abstract}

\section{Keywords}

Camera sensor networks, Reaction diffusion, Biological system

\section{INTRODUCTION}

Sensor network is one of the most promising and key technologies for safe, secure, and comfortable society. By distributing a large number of sensor nodes and organizing a network through wired and/or wireless communication, one can obtain detailed information about surroundings, remote region, and objects. In particular, camera sensor networks, which are composed of nodes equipped with a camera, are useful in a variety of applications such as remote surveillance, home security, and tracking applications [1].

Generally, control mechanisms for sensor networks must be scalable, adaptive, and robust, because of a large number of sensor nodes, random or unplanned deployment, and

Permission to make digital or hard copies of all or part of this work for personal or classroom use is granted without fee provided that copies are not made or distributed for profit or commercial advantage and that copies bear this notice and the full citation on the first page. To copy otherwise, to republish, to post on servers or to redistribute to lists, requires prior specific permission and/or a fee.

Bionetics'07 December 10-13, 2007, Budapest, Hungary

Copyright 2007 ICST 978-963-9799-11-0. dynamic topology changes due to addition, movement, and removal of sensor nodes. In addition, due to difficulty in managing a large number of nodes in a centralized manner, mechanisms must be fully distributed and self-organizing [2]. Moreover, a particular problem for camera sensor networks is transmission of high volume traffic of video data over a wireless network of limited capacity. Therefore, in sending video data to data gathering centers in real-time, nodes must adjust the video coding rate taking into account the network capacity and the importance of video data. In a case of surveillance or monitoring applications, video data obtained at a camera, which detects and tracks a suspicious or interesting object, should have the sufficiently high resolution and high quality to have a better look at the target.

To accomplish the above mentioned goals, we adopt a reaction-diffusion model in this paper. A reaction-diffusion equation was first proposed by Alan Turing as a mathematical model for pattern generation on the surface of body of fishes and mammals [6]. In a reaction-diffusion model, through local interactions among molecules of neighboring cells, a variety of patterns of morphogen concentrations emerge in a self-organizing manner. Autonomously generated patterns can be used for routing, clustering, scheduling, and topology control on sensor networks [3, 4, 5, 7].

In this paper, we propose an autonomous mechanism based on a reaction-diffusion model for coding rate control in camera sensor networks for remote surveillance and tracking applications. Specifically, we generate spot patterns so that the morphogen concentrations have a peak at a camera detecting a target in its observation area. In addition, to prepare cameras in the direction of a moving target for future detection, their video coding rates, in other words, their morphogen concentrations are made slightly higher than others. As a result, we see a pattern of concentric circles for a stationary target and an elliptic pattern for a moving target. Furthermore, we also propose a mechanism to keep the total of coding rate low for closely located targets considering the limited capacity of a wireless network.

The rest of the paper is organized as follows. In Section 2, we describe our reaction-diffusion based control mechanism for camera sensor networks. In Section 3, we then show and discuss results of simulation experiments. Finally, we conclude the paper in Section 4. 


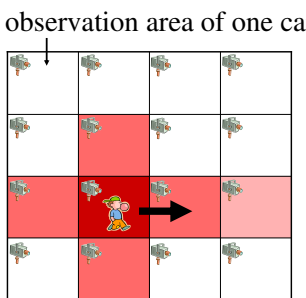

Figure 1: Camera sensor network

\section{REACTION-DIFFUSION BASED CONTROL OF CAMERA SENSOR NETWORKS}

Figure 1 illustrates a surveillance or monitoring system that we consider in this paper. Each square corresponds to the observation area of a camera sensor node. The darker the square is, the higher the video coding rate is. We assume that nodes are arranged in a grid topology, considering town or room monitoring as an application of the mechanism. A node can communicate with four neighbors in up, right, down, and left directions. The assumption on node layout can be relaxed by adapting a numerical solution technique for $\nabla^{2}$ (details are not shown for space limitation).

Each node has a camera and a wireless communication device. A camera or a node has the capability of object recognition and motion detection with which the existence, speed, and direction of a target in its observation area.

\subsection{Basic behavior}

Basically, at regular control intervals of $T$ seconds, each node calculates the reaction-diffusion equation by using the information it received in the preceding control interval, adjusts its video coding rate in accordance with morphogen concentrations, and then broadcasts a message containing information about its morphogen concentrations, stimulus $E$, stimulus direction, attenuation coefficient $A$, and NIP (Notification of Inhibitor Peak) notification to its neighbors. We call the duration between the $t$-th control timing and the $t+1$-th control timing as the $t$-th control interval. We should note here that timing of reaction-diffusion calculation and message emission is different among nodes, although the control interval is identical among nodes.

The reaction-diffusion equation is identical among nodes.

$$
\left\{\begin{array}{l}
\frac{\partial u}{\partial t}=F(u, v)+D_{u} \nabla^{2} u+E(t) \\
\frac{\partial v}{\partial t}=G(u, v)+D_{v} \nabla^{2} v,
\end{array}\right.
$$

where $u$ and $v$ are concentrations of activator and inhibitor, respectively. $D_{u}$ and $D_{v}$ are the diffusion rate of activator and inhibitor, respectively. $E(t)$ is the amount of stimulus. The first term of the right-hand side is a reaction team and the second term is a diffusion term. $F$ and $G$ are nonlinear functions for reactions and are described as follows,

$$
\left\{\begin{array}{l}
F(u, v)=\max \{0, \min \{a u-b v-c, M\}\}-d u \\
G(u, v)=\max \{0, \min \{e u-h v-f, N\}\}-g v .
\end{array}\right.
$$

Since nodes are arranged in a grid layout and messages are exchanged at regular intervals, we spatially and temporally discretize Eqs. (1) and (2) as follows.

$$
\left\{\begin{aligned}
u_{t}= & u_{t-1}+\Delta t\left\{F\left(u_{t-1}, v_{t-1}\right)+E(t-1)\right. \\
& \left.+D_{u} \frac{\left(u_{t-1}^{u}+u_{t-1}^{d}+u_{t-1}^{l}+u_{t-1}^{r}-4 u_{t-1}\right)}{\Delta h^{2}}\right\} \\
v_{t}= & v_{t-1}+\Delta t\left\{G\left(u_{t-1}, v_{t-1}\right)\right. \\
& \left.+D_{v} \frac{\left(v_{t-1}^{u}+v_{t-1}^{d}+v_{t-1}^{l}+v_{t-1}^{r}-4 v_{t-1}\right)}{\Delta h^{2}}\right\}
\end{aligned}\right.
$$

$\left\{\begin{array}{l}F\left(u_{t-1}, v_{t-1}\right)=\max \left\{0, \min \left\{a u_{t-1}-b v_{t-1}-c, M\right\}\right\}-d u_{t-1} \\ G\left(u_{t-1}, v_{t-1}\right)=\max \left\{0, \min \left\{e u_{t-1}-h v_{t-1}-f, N\right\}\right\}-g v_{t-1} .\end{array}\right.$

At the $t$-th control timing, a node calculates the above equation to derive its morphogen concentrations $u_{t}$ and $v_{t}$. A set of $u_{t-1}^{u}, u_{t-1}^{d}, u_{t-1}^{l}$, and $u_{t-1}^{r}$ and a set of $v_{t-1}^{u}, v_{t-1}^{d}, v_{t-1}^{l}$, and $v_{t-1}^{r}$ correspond to concentrations of activator and inhibitor of neighboring nodes in up, down, left, and right directions. These values are obtained from messages received in the $t-1$-th control interval. If a node did not receive a message from a neighboring node in the $t-1$-th control interval, the latest value obtained in the preceding intervals is used instead. $\Delta h$ and $\Delta t$ correspond to the distance between nodes and the discrete step interval of time, respectively.

$E(t-1)$ in Eq. (3) is the amount of stimulus which is determined at the $t$-th control timing, based on messages it received in the $t-1$-th control interval and the condition of a target if exists. The stimulus controls the distribution of morphogen concentrations, that is, a pattern. Usually, the amount of stimulus is zero. A node which detects a target in the $t-1$-th control interval appropriately sets $E, A$, and stimulus direction in accordance with the velocity of the target, so that a spot pattern centered at the node is generated. The stimulus diffuses to nodes in the stimulus direction, which is the same direction of target movement. The attenuation coefficient $A$ is used for this purpose. Details of stimulus determination and diffusion will be explained in subsection 2.2. NIP notification is used to regulate the amount of stimulus when two or more targets are closely located. Details of NIP will be given in subsection 2.3.

Once morphogen concentrations are derived, a node translates the concentrations to the video coding rate that it uses during the $t$-th control interval. A spatial pattern of a reaction-diffusion model comes from the spatial heterogeneity in concentration of activator. Since the region between two closely-located peaks of the concentration of activator has the high concentration of activator, in our mechanism, by focusing on a phenomenon that the concentration of inhibitor is also high in the region, a node determines the video coding rate based on $u / \sqrt{v}$. If $v<0.001$, then we set $u / \sqrt{v}=0$, in order to avoid the divergence of $u / \sqrt{v}$. In the paper, we use the value $u / \sqrt{v}$ as the video coding rate.

\subsection{Stimuli arrangement}

If a node has a target in its observation area at the control timing, it sets the amount of stimulus $E$, the attenuation coefficient $A$, and the stimulus direction. The node calculates the morphogen concentrations, set the video coding rate, and broadcasts a message containing this information. A node receiving a message first sees whether it is in the stim- 


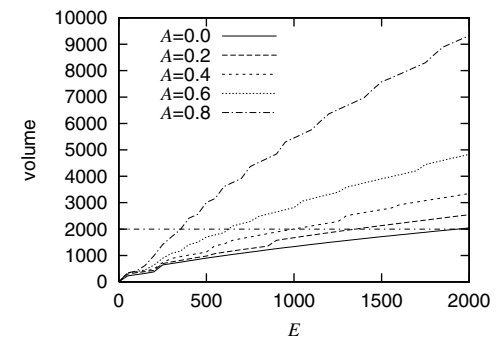

Figure 2: Relationship among $A, E$, volume of pattern

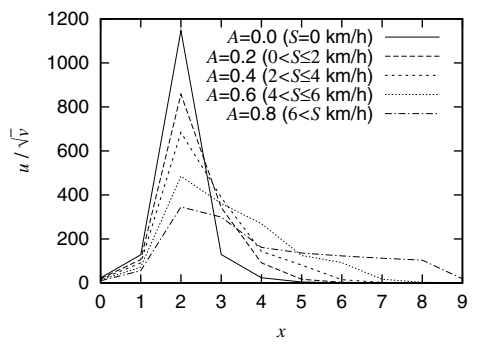

Figure 3: Distribution of $u / \sqrt{v}$
Table 1: Mapping from $S$ to $A$ and range of $E$

\begin{tabular}{|c|c|c|c|}
\hline$S(\mathrm{~km} / \mathrm{h})$ & $A$ & $E_{\max }$ & $E_{\min }$ \\
\hline$S=0$ & 0.0 & 1960 & 830 \\
\hline $0<S \leq 2$ & 0.2 & 1370 & 700 \\
\hline $2<S \leq 4$ & 0.4 & 1010 & 440 \\
\hline $4<S \leq 6$ & 0.6 & 620 & 390 \\
\hline $6<S$ & 0.8 & 360 & 260 \\
\hline
\end{tabular}

ulus direction. If not, it ignores the information. If it is, it first calculates the amount of stimulus $E^{\prime}$ from the informed $E$ and $A$ as $E^{\prime}=A \times E$. This stimulus is used as $E(t-1)$. After the calculation, the information about the stimulus, including $E^{\prime}, A$, and the direction is further diffused to neighbor nodes by being embedded in a broadcast message. When a node receives multiple messages containing stimuli from neighbor nodes, it uses the maximum $E^{\prime}$ as $E(t-1)$.

The amount of stimulus $E$ and the attenuation coefficient $A$ are determined in accordance with the speed of target and the capacity of wireless channel. In Fig. 2, the relationship among $E, A$, and the volume of a generated pattern is shown for the case with a single target. The volume of a pattern is defined as,

$$
V(E, A)=\sum_{(i, j)} u(i, j) / \sqrt{v(i, j)} .
$$

$u(i, j)$ and $v(i, j)$ are the concentrations of activator and inhibitor at node $(i, j)$ on a converged stable pattern, respectively. Since a node chooses the video coding rate depending on $u(t) / \sqrt{v(t)}$, the volume corresponds to the total amount of traffic generated by nodes in a spot. Since the attenuation coefficient $A$ determines the range of stimulus diffusion, a node detecting a target first sets the attenuation coefficient $A$ in accordance with the speed $S$ of target. Then, the node determines the stimulus $E$ from $V(E, A)$ in order to keep the total traffic at a certain volume. For example, when the capacity of local wireless network is 2000 in volume and $A$ is $0.4, E$ is set at 1010 . The mapping from the network capacity to the volume and from the speed to the attenuation coefficient depends on the system conditions and application requirements.

\subsection{Stimuli adjustment}

When two or more targets are located close together, they activate each other and the total amount of video traffic would exceed the network capacity in that area. To tackle the problem, we additionally propose the stimuli adjustment mechanism. A basic idea is as follows. When two or more targets are close together, both of concentrations of activator and inhibitor become high at the inbetween region. Especially, the concentration of inhibitor has a peak at the center. By using this phenomena, a node at the overlapping point detects the overlap.

At the control timing, a node compares the concentration of inhibitor of itself with those of neighboring nodes. If its concentration is the highest and it does not have a target in its observation area, it sets the NIP notification in a message it broadcasts. Now, a node receives a message. If $u / \sqrt{v}$ of the node is higher than that of a node from which it received NIP, the node has the stimulus, or any neighboring node of the node has a target, it sets NIP in a broadcast message. As a consequence, NIP follows the gradient of $u / \sqrt{v}$ and diffused stimuli toward nodes having a target.

When a node having a target receives a message with NIP, it reduces the stimulus $E$ as $E \times \alpha(0<\alpha<1)$. When the targets move apart from each other and the overlap disappears, the stimulus has to be increased. Therefore, a node having a target increases the stimulus as $E=E+\Delta e$, if it does not receive any NIP in the preceding control interval. The stimulus must be large enough to generate a pattern and smaller than the maximum to keep the volume. The range $E_{\min } \leq E \leq E_{\max }$ is determined from $V(E, A)$. For example, with $A=0.4$, the range is from 440 to 1010 .

\section{SIMULATION EXPERIMENTS}

In this section, we show results of simulation experiments to verify the effectiveness of our mechanism. One hundred nodes are arranged in a $10 \times 10$ grid with separation of 100 meters. Parameter setting for the reaction-diffusion equation is, $a=0.08, b=0.2, c=0.2, d=0.03, e=0.1$, $f=0.05, g=0.14, h=0.06, D_{u}=0.004, D_{v}=0.1$, $M=0.2, N=0.5, \Delta t=0.1$, and $\Delta h=1$. For the stimuli adjustment, $\alpha=0.999$ and $\Delta e=1$ are used. Assuming that the local capacity of wireless network is 2000 in volume, the mapping from the speed $S$ to the attenuation coefficient $A$ and the range of the stimulus $E$ are summarized in Table 1. Initially, the morphogen concentrations are set at zero.

First, we set the speed of a stationary node at $(2,5)$ as 1 , 3,5 , and $7 \mathrm{~km} / \mathrm{h}$. Figure 3 shows the distribution of $u / \sqrt{v}$ for the different attenuation coefficient on $y=5$. As can be seen, as $A$, i.e., the speed of moving target, increases, the resultant pattern spreads wider and the height of peak becomes lower. The total volume is kept constant at lower than 2000 in Fig. 3.

Now, we consider a scenario with multiple moving targets. Initially, targets which move to the random direction at the random speed from 0 to $8 \mathrm{~km} / \mathrm{h}$ are located at randomly chosen nodes. At every control timing, a target changes the speed and direction with the probability of 0.005 . The control interval is set at 2 seconds. At every $180 / \mathrm{V}$ control intervals, a moving target migrates to an observation area of neighboring node in the moving direction. The discrete step $\Delta t$ is set at 2.0 .

Figure 4(a) depicts the transition of volume with three moving targets. Depending on the distance among targets 


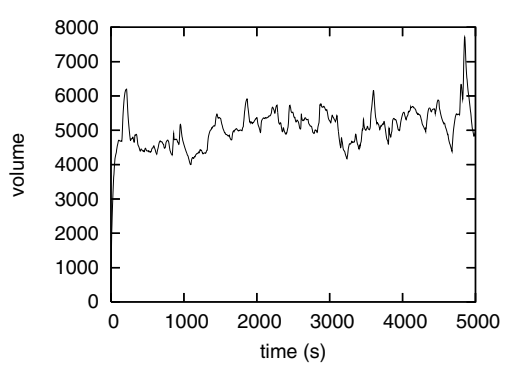

(a) Transition of volume with three moving targets

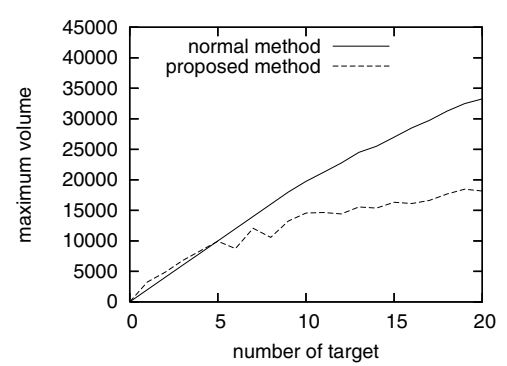

(b) Maximum volume against number of moving targets

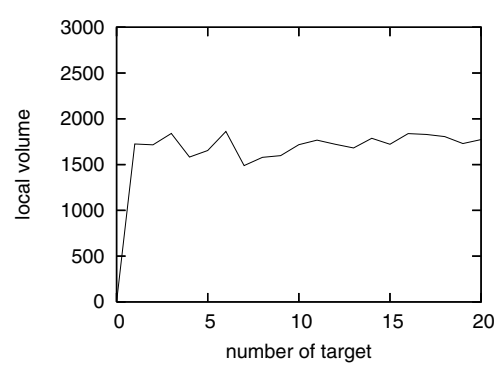

(c) Local volume against number of moving targets

Figure 4: Simulation results of multiple moving targets

and their speed and direction, the total volume dynamically changes. One reason that the total volume exceeds 6000 , i.e., the triple of the volume of a single target, is that the high concentration of activator sometimes remains behind a fast moving target. The other reason is that suppression of stimulus $E$ is too slow for closely located targets moving at the speed of more than $6 \mathrm{~km} / \mathrm{h}$. We need to accelerate pattern adaptation, but it is one of future works.

Figure 4(b) show the relationship among the number of targets and the maximum volume of generated patterns. The solid line stand for the normal method, which set the video coding rate as 1000 at the node that detects a target, 250 at the neighboring nodes. As shown in the figures, our mechanism suppresses the traffic volume much lower by reaction-diffusion based control.

Figure 4(c) show the local volume against number of moving targets. The local volume is the total of coding rate of five nodes at the center of area. From this figure, we can see that the proposed method keeps the local volume under the local network capacity independently of the number of targets. From Fig. 4(b) and Fig. 4(c), we can see that the proposed method suppresses the total coding rate in the whole area, while keeping the high coding rate in local area.

Although results are not shown, we also conducted simulation experiments on a large-size network, where 2500 nodes are arranged in a $50 \times 50$ grid with separation of 100 meters. We verified that every node appropriately adjusts the video coding rate to monitor moving targets.

\section{CONCLUSIONS}

In this paper, we proposed a reaction-diffusion based autonomous control mechanism for camera sensor networks. In our mechanism, nodes periodically exchange information about the morphogen concentrations, calculate the reactiondiffusion equation, and adjust the video coding rate. By setting the stimulus at a node detecting a target and diffusing the stimulus, the video coding rate becomes high at a node with a target and nodes in the moving direction.

Although the effectiveness of the mechanism is verified through simulation experiments, we require further improvement. Since the video traffic is concentrated in the proximity of a target, there is the possibility of severe congestion in the local wireless network even with the coding rate control. We are going to incorporate control mechanisms of multi- ple layers with coding rate adjustment. We now consider a reaction-diffusion based mechanism to mitigate congestion caused by video traffic.

\section{ACKNOWLEDGMENTS}

We are very grateful to Assistant Professor Etsushi Nakaguchi for his valuable cooperation in our research. This research was partly supported by "Special Coordination Funds for Promoting Science and Technology: Yuragi Project" of the Ministry of Education, Culture, Sports, Science and Technology, Japan.

\section{REFERENCES}

[1] I. F. Akyildiz, T. Melodia, and K. R. Chowdhury. A Survey on Wireless Multimedia Sensor Networks. Computer Networks, 51(4):921-960, Mar. 2007.

[2] H. Chan and A. Perrig. ACE: An Emergent Algorithm for Highly Uniform Cluster Formation. Proceedings of the First European Workshop on Sensor Networks (EWSN), pages 154-171, Jan. 2004.

[3] Y. Chen and T. C. Henderson. S-nets: Smart Sensor Networks. In Proceedings of International Symposium on Experimental Robotics, pages 85-94, Dec. 2000.

[4] M. Durvy and P. Thiran. Reaction-Diffusion Based Transmission Patterns for Ad Hoc Networks. In Proceedings of IEEE INFOCOM 2005, volume 3, pages 2195-2205, Mar. 2005.

[5] K. Hyodo, N. Wakamiya, E. Nakaguchi, M. Murata, Y. Kubo, and K. Yanagihara. Experiments and Considerations on Reaction-Diffusion based Pattern Generation in a Wireless Sensor Network. In Proceedings of 1st IEEE International Workshop: From Theory to Practice in Wireless Sensor Networks (t2pWSN 2007), Jun. 2007.

[6] A. M. Turing. The Chemical Basis of Morphogenesis. Royal Society of London Philosophical Transactions Series B, 237:37-72, Aug. 1952.

[7] A. Yoshida, K. Aoki, and S. Araki. Cooperative Control Based on Reaction-Diffusion Equation for Surveillance System. In Proceedings of Ninth International Conference on Knowledge-Based Intelligent Information and Engineering Systems, pages 533-539, Sep. 2005. 